\title{
Percepción, consumo y factores asociados con el fenómeno droga en población escolar de Heredia, Costa Rica
}

\author{
Obando Solano, P.*; Sáenz Rojas, M.A.** \\ * Investigadora-Antropóloga. Instituto sobre Alcoholismo y Farmacodependencia. Profesora Universidad de Costa Rica \\ * Asesor Psicosocial. Dirección Nacional de Prevención de la Violencia y el Delito del Ministerio de Justicia y Gracia. \\ Profesor Universidad Centroamericana de Ciencias Sociales.
}

\section{Resumen}

Se analiza el uso y abuso de drogas, así como factores protectores y de riesgo en el tema de la drogadicción en niños escolarizados entre los 9 y 14 años de edad en el nivel nacional. Se obtuvieron datos de tres escuelas primarias urbano-marginales de la provincia de Heredia. Un total de 386 estudiantes fueron entrevistados mediante un cuestionario autoadministrado.

Se detectó una edad de inicio de consumo más temprana en comparación con la población nacional, especialmente para alcohol. El alcoholismo y el abuso de drogas son considerados como los principales problemas de la comunidad y se reportó un consumo excesivo de alcohol en el grupo familiar, especialmente por parte del padre, lo que podría influir en la imitación de patrones de comportamiento y consumo del niño. Otro factor de importancia es el hecho que un $36 \%$ de los niños provienen de familias uniparentales, de las cuales el $40 \%$ de las madres son tomadoras excesivas de alcohol.

Existe una significativa correlación entre la oferta y el consumo de drogas en los niños que reportaron haber consumido alguna vez en su vida, en donde al $60 \%$ le ha sido ofrecida en contexto hogareño. La religión y la información sobre los efectos de la droga juegan un papel de protección importante.

Palabras clave: Consumo de drogas, niños, escuela, alcohol, tabaco, drogas ilegales.

\begin{abstract}
This study analyzes drug use, abuse, risk and protective factors associated to the drug issue among children aged 9 to 14 years all across the country.

Data from 3 primary suburban schools located in one of the seven provinces of the country is recorded and analyzed for the purpose of this paper. A total of 384 school children were interviewed using a self-administered 22 items questionnaire. In general, an early age of onset, especially for alcohol use was found for this population, finding no gender differences. Alcoholism and drug abuse are considered the main problems faced by this community. Alcohol abuse is been reported, mainly by the father, which could be potentially assimilated by the children, thinking of a drinking behavioral pattern as well as another drug use patterns. Another factor which should be considered is that $36 \%$ of these children, come from single parenting homes, where $40 \%$ of the mothers are heavy alcohol drinkers.

A significant correlation was found between drug offer and drug use among those kids who reported having used drugs sometime in their life. Furthermore, $60 \%$ of these children have been offered drug in their own household. Protective factors were identified, mostly related to religion and health information.
\end{abstract}

Key Words: Drug abuse, children, school, alcohol, tobacco, ilegal drugs.

\section{INTRODUCCIÓN}

a atención en los ámbitos de la salud y la educación de la niñez y, en general, el mejoramiento relativo de las condiciones materiales de existencia de la población, fueron aspectos significativamente favorecidos mediante diversas políticas sociales desarrolladas por el Estado costarricense hasta inicios de la década de 1980.

Por otra parte, la crisis económica mundial que marcó el comienzo de esa década y las políticas derivadas de los programas de ajuste estructural, produjeron un deterioro de grandes proporciones en la calidad de vida de nuestro país. Estas condiciones aunadas al 
incremento del tráfico de drogas desde Sudamérica hacia Norteamérica y Europa, así como a la práctica de asumir estilos de vida basados en el consumismo, propios de los países industrializados, y el incremento del turismo internacional, resultan en probables explicaciones sobre la situación de consumo de drogas ilícitas y el aumento en la ingesta de sustancias legalizadas a lo largo del territorio nacional.

A pesar de lo anterior, se ha carecido de investigación sobre el consumo de sustancias psicoactivas en población infantil que cursa la enseñanza general básica, lo cual se deriva, en parte, de los hallazgos de múltiples estudios efectuados en el país (Herrera y Valerio, 1977; Míguez, Bolaños y Alfaro, 1985; (Bejarano, Amador y Vargas, 1994). Estos han demostrado que, a excepción de las sustancias inhalables, para las restantes drogas el inicio en el consumo se produce después de los 12 años de edad, momento en el que generalmente los/as niños/as han concluido la educación primaria.

La investigación llevada a cabo en Costa Rica con este tipo de población, ha centrado su atención en la minoridad inhaladora de sustancias volátiles ("thinner", pegamento, gasolina, entre otras), generalmente niñas y niños procedentes de sectores urbano-marginales, con un sensible déficit en su nivel educativo (Guzmán, 1982; Míguez, 1984; Alfaro, Cerdas e Ibarra, 1992), o en niños y adolescentes detenidos en el único centro de orientación juvenil para menores infractores existente en el país (Míguez, 1983).

Además, dos elementos merecen destacarse: por un lado, en Costa Rica no se han efectuado estudios estructurados dirigidos a establecer la magnitud del consumo de drogas legales o ilegales en sujetos en edad escolar (a excepción de tres aproximaciones previas adscritas a este mismo proyecto), y que contemplen sus percepciones sobre aspectos generales de tales sustancias; por otra parte, diferentes fuentes, tanto privadas como oficiales, han destacado en años recientes la importancia de conocer la realidad de este grupo poblacional, acerca del que existe poca información y sobre el que debiera enfocarse parte de los programas de prevención.

Esta situación cobra mayor relevancia al tomar en consideración la importancia que las últimas administraciones de gobierno han asignado a las poblaciones en condiciones socioeconómicas precarias y en riesgo social. Entre ellas se encuentran los/as niños/as de centros educativos urbano-marginales. Aunado a lo anterior los estudios en poblaciones infantiles se han planteado como una de las prioridades de investigación del Instituto sobre Alcoholismo y Farmacodependencia y de la Comisión Regional Inter-Institucional de Prevención Integral del Fenómeno Droga (CRIPI) de Heredia. Adicionalmente, están las percepciones de docentes y miembros de estas comunidades, quizás influidas de manera significativa por los medios de difusión, las cuales indican una realidad de consumo de drogas en escuelas que no ha sido detectada. En virtud de lo anterior, el presente trabajo es un intento de aproximación y sistematización de las opiniones y el consumo de drogas en niños/as de $5^{\circ}$ y $6^{\circ}$ grados de escuelas urbano-marginales en la Región de Enseñanza de Heredia.

Territorialmente, Heredia es la provincia más pequeña de Costa Rica; sin embargo, relativamente es la tercera provincia más poblada del país, con una distribución de 107 habitantes por km², localizada en una extensión de $2.673,49 \mathrm{~km}^{2}$, en un país que cuenta con 51,100 km². Para 1998 Costa Rica poseía una tasa de ocupación del 52,2\% y una tasa de desempleo abierto del $5,6 \%$, con un $19,7 \%$ de hogares pobres.

El presente estudio se propone los siguientes objetivos: a.- analizar las características del consumo de sustancias psicoactivas y la percepción hacia éstas, de los/as niños/as de $5^{\circ}$ y $6^{\circ}$ grados de tres escuelas urbano-marginales de Heredia y b.- identificar factores de riesgo y protectores asociados con el consumo de drogas en los/as niños/as estudiados/as que coadyuven en la formulación de estrategias preventivas dirigidas a ese sector poblacional. Asimismo, pretende servir de base para el desarrollo de investigaciones específicas que profundicen los hallazgos y promuevan acciones de intervención propias para cada comunidad o localidad.

\section{MATERIAL Y MÉTODO}

\subsection{Sujetos:}

Se encuestó a un total de 386 escolares de $5^{\circ}$ y $6^{\circ}$ grados de enseñanza primaria de ambos sexos, con un rango de edad de los 9 a los 14 años. En este sentido, se trabajó en tres escuelas urbano-marginales localizadas en el cantón central de la provincia de Heredia (Guararí, Los Nísperos o La Milpa y La Gran Samaria), lo que representa un $50 \%$ del total de escuelas urbano-marginales y un $7 \%$ de la totalidad de escuelas de la provincia, las cuales fueron seleccionadas por tener una densidad de población estudiantil mayor y pertenecer a comunidades con importantes deficiencias socioeconómicas.

Para la realización de este estudio no se requirió ningún procedimiento de muestreo convencional, ya que se utilizó una metodología censal, es decir, se aplicó el instrumento al total de estudiantes presentes en las aulas.

\subsection{Instrumento:}

Los datos fueron obtenidos mediante la aplicación de un cuestionario compuesto de 22 preguntas, el cual fue diseñado por personal de la unidad de Investigación 
del Instituto sobre Alcoholismo y Farmacodependencia (IAFA). Dicho cuestionario ha sido validado mediante diversos procedimientos, tales como revisión interna, revisión de expertos y prueba piloto; asimismo, se ha utilizado desde 1996 con propósitos similares al de la presente investigación en escuelas públicas de las provincias de Guanacaste, Puntarenas y Limón.

El cuestionario fue anónimo, lo cual se reiteró a los y las estudiantes previo al inicio de la aplicación, y señalado en la primera página del mismo.

El instrumento fue diseñado tomando en consideración 4 áreas de interés:

-Información sociodemográfica general

-Percepción del ambiente del estudiante y de sí mismo

-Percepción de riesgo de consumo

- Consumo de drogas

El instrumento se aplicó de manera grupal por parte de tres estudiantes de último año de la carrera de Psicología, quienes fueron debidamente entrenados. Dicha labor se efectuó durante el mes de noviembre de 1998 en las tres escuelas seleccionadas, previa coordinación entre la Comisión Regional InterInstitucional de Prevención Integral (CRIPI) de Heredia y la Dirección Regional de Enseñanza. Los datos fueron procesados mediante el programa estadístico SPSS versión 8.0, y se analizaron el estadístico $x^{2}$ y la correlación de Pearson r, con un nivel de significancia igual o menor al .05.

\subsection{Características socio-demográficas de la po- blación:}

Tal como se aprecia en la tabla 1, la proporción de la población según el sexo es relativamente similar. La mayoría de niños y niñas se ubicaron entre los 11 y 12 años de edad (59\%), intervalo esperable para estos grados escolares, registrándose en la población estudiada un promedio de edad de 12,3 años; asimismo, el hecho que más de una tercera parte de la población estudiada se ubicara entre los 13 y 14 años (39\%), remite a la posibilidad que un amplio sector se trate de estudiantes con dificultades académicas que por diversos motivos no han podido cumplir con la rígida estructura de la educación formal.

Por otra parte, la mitad de los casos refirió proceder de una familia nuclear, mientras que una tercera parte manifestó convivir solamente con su madre y hermanos/as, situación que refleja el fenómeno social de las madres jefas de hogar. En lo que respecta al aporte del ingreso al hogar se debe resaltar la importancia del papel de la mujer y su incorporación al proceso productivo, reafirmándose el planteamiento anterior en lo relativo a su rol como principal proveedora económica (Tabla 1.).
Tabla 1. Características socio-demográficas de estudiantes de $5^{\circ}$ y $6^{\circ}$ grados de escuelas urbano-marginales: Heredia, Costa Rica, 1998.

\section{Variable}

Porcentaje

\section{Sexo:}

-Masculino $\quad 51,3$

$\begin{array}{ll}\text {-Femenino } & 48,7\end{array}$

Edad:

$-09-10$

$-11-12$

59,1

$-13-14$

39,1

Grado:

-5 to.

52,3

-6to.

Grupo Familiar:

-Ambos padres/hermanos 49,0

-Padre/hermanos $\quad 5,2$

-Madre/hermanos $\quad 32,1$

-Sólo con padre $\quad 1,0$

-Sólo con madre $\quad 5,7$

-Otro/a pariente 7,0

Trabaja el niño/a y le pagan:

-Sí

-No

96,4

Aporte del ingreso al hogar:

-Sólo padre 38,3

-Sólo madre $\quad 21,2$

-Ambos padres 23,6

-Hermanos/as $\quad 2,6$

-Hermanos/as junto a uno o ambos padres 9,3

-Otro 3,1

-Madre/otro $\quad 0,8$

-Padres/otro 0,3

-Ambos padres/hermanos/as y otro $\quad 0,5$

$-\mathrm{N} / \mathrm{R}$

0,3

Religión:

-Católica $\quad 58,8$

-Cristiano-protestante 22,8

-Otra $\quad 9,1$

-Ninguna $\quad 9,1$

-N/R $\quad 0,3$

Si bien la proporción de menores que expresaron trabajar y devengar un pago por su trabajo fue bastante reducida $(3,1 \%)$, es necesario señalar que la legislación costarricense establece prohibición de trabajar a menores de 15 años de edad ${ }^{1}$.

Otro elemento que se destaca es que alrededor del $90 \%$ de la población estudiada indicó mantener algún credo religioso, aspecto que luego será retomado en el estudio de factores asociados con el consumo de drogas.

\footnotetext{
' Ley Nº 7739 de 6 de febrero de 1998, "Código de la Niñez y la Adolescencia" (artículo 92)
} 


\section{RESULTADOS}

\subsection{Percepción social:}

En torno al principal problema de su comunidad, el grupo en estudio proporcionó diferentes opiniones, las cuales se pueden apreciar en la tabla 2.

De lo anterior se resalta el alcoholismo y el consumo de drogas como dos de los principales problemas percibidos en su comunidad.

Además, un $44,2 \%$ de la población estudiada reportó haber escuchado "mucho" acerca de las drogas, contra un $26,2 \%$ que indicó "poco", mientras que un $29,5 \%$ señaló "regular".

Tabla 2. Opiniones de estudiantes de $5^{\circ}$ y $6^{\circ}$ grados de escuelas urbano-marginales sobre el principal problema de su comunidad: Heredia, Costa Rica, 1998.

\begin{tabular}{lc}
\hline Variable & Porcentaje \\
\hline Delincuencia & 46,6 \\
Alcoholismo & 44,3 \\
Consumo de drogas & 36,0 \\
Contaminación ambiental & 29,0 \\
Tráfico de drogas & 13,0 \\
Falta de vivienda & 12,4 \\
Ningún problema & 11,1 \\
Prostitución & 4,7 \\
Otros problemas & 0,5 \\
\hline
\end{tabular}

Las diferencias entre la percepción sobre la droga de la que más han escuchado y la que consideran más peligrosa (ver figura 1), remiten a cuestionarse sobre la pertinencia de la información con contenido educativo, publicitario, noticioso y popular recibida por los y las estudiantes, muchas veces mediatizada por el efecto distorsionador de los medios de difusión de masas. Asimismo, llama la atención que si bien el alcoholismo es considerado el segundo problema en importancia en la comunidad, no es considerado tan peligroso como lo son otras drogas. Esta diferencia de percepción se observó en otras poblaciones escolares del mismo rango de edad (Obando, Bejarano y Ugalde, 1998), así como en la población general durante la encuesta nacional sobre consumo de drogas de 1995 (Bejarano, Carvajal y San Lee, 1996).

Esta peligrosidad asignada a las drogas ilegales explica parcialmente el alto consumo de drogas legales como droga de inicio por parte de esta población. Lo anterior demuestra la importancia de contemplar el nivel informativo sobre los efectos de la droga en los programas de prevención, a pesar de que se ha establecido que los componentes cognocitivos no son suficientes para cambiar la actitud hacia el consumo de drogas.

Además, investigaciones nacionales (Bejarano, Amador y Vargas, 1994; Sáenz et al, 1998) han encontrado que las personas tienden a asignarle a los patrones más frecuentes de consumo un mayor riesgo para la salud.

Figura 1. Criterio de estudiantes de $5^{\circ}$ y $6^{\circ}$ grados de escuelas urbano-marginales sobre droga más escuchada y más peligrosa: Heredia, Costa Rica, 1998 (Valores porcentuales)

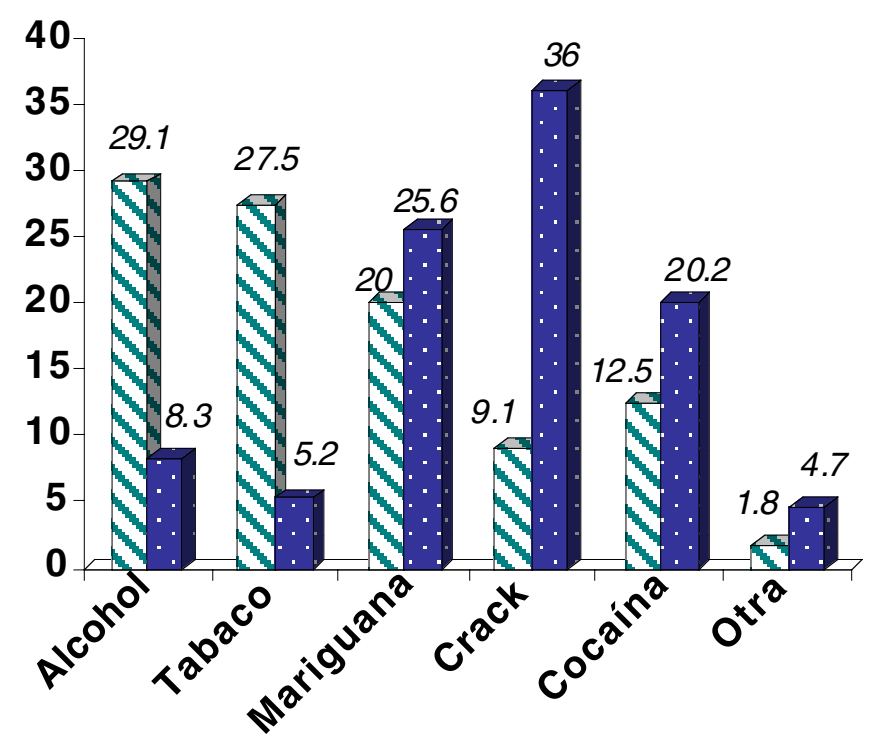

Droga más escuchada:॥ Droga más peligrosa 


\subsection{Consumo en la familia:}

En la tabla 3 se presentan las proporciones de ingesta excesiva de alcohol y consumo de drogas ilegales en miembros de la familia.

Los valores obtenidos pueden estimarse relativamente bajos, sobre todo al considerar, por una parte, la zona de residencia, la cual tradicionalmente se ha vinculado con significativos problemas asociados con el fenómeno droga en general y, por otra, la opinión de la población estudiada en torno al principal problema de su comunidad (datos planteados en la tabla 2). Estos niveles de consumo reportados podrían obedecer, en alguna medida, a la idealización que las/os niñas/os mantienen de sus figuras parentales hasta los inicios de la adolescencia. (Tabla 3)

No obstante, es de importancia señalar que la mitad de las y los estudiantes $(49,6 \%)$ toman como modelos en sus vidas a alguno/a de sus parientes, al indicar que les gustaría parecerse a ellos/as. Esta situación podría favorecer la imitación de patrones de comportamiento y de consumo significativos en los/as niños/as, propios de los/as adultos/as, aunado a que diversas investigaciones (Sandí et al., 1995) han reconocido algunos aspectos relacionados con el ámbito familiar como factores de riesgo asociados con el consumo de drogas. En efecto, de aquellos estudiantes que admitieron haber consumido alguna droga, el $43,4 \%$ tuvo como modelo a alguien de la familia, proporción que resulta llamativa y sugiere la necesidad de estudios más profunda, pues no es estadísticamente significativa.
Igualmente, el $40 \%$ de los/as niños/as que han consumido conviven con un grupo familiar nuclear (padre, madre y hermanos/as) y un $36 \%$ convive con la madre y sus hermanos/as. Este dato recalca el impacto que tiene, tanto la estructura familiar como los patrones que ahí se desarrollen, sobre los patrones de consumo y comportamiento del niño/a.

En otras palabras, un $76 \%$ de los niños consumidores conviven con por lo menos un padre, esto refleja una debilidad en la supervisión y control social hacia los niños por parte de los padres, en la medida que esto se constituyen en las principales figuras de autoridad durante el proceso de socialización. Asimismo, la imitación del comportamiento en relación con los patrones de consumo de la familia, junto al ambiente permisivo presente, facilita que los/as niños/as registren los niveles de ingesta reportados anteriormente.

En su mayoría (89\%), los miembros económicamente activos/as del grupo familiar con que convive el niño o la niña, se dedican a actividades remunerativas en los sectores secundario (sector industrial) y terciario (servicios públicos y profesionales). Es en estos sectores donde se encuentra, además, el $90 \%$ de los/as niños/as que han consumido, junto al $68,4 \%$ de los padres y las madres que consumen excesivamente alcohol y el $100 \%$ de los/as que consumen drogas ilegales. Curiosamente, para el caso de la madre, el $40 \%$ de las que ingieren excesivamente alcohol son amas de casa, dato que coincide con el de otras investigaciones de carácter nacional (Bejarano, Carvajal y San Lee, 1996; Bejarano, San Lee y Carvajal, 1999; Jiménez y Bejarano, 1991), el resto se distribuye entre el sector secundario y el terciario.

Tabla 3. Consumo de alcohol y drogas ilícitas en familiares de estudiantes de $5^{\circ}$ y $6^{\circ}$ grados de escuelas urbano-marginales, por relación de parentesco, según sustancia: Heredia, Costa Rica, 1998 (Valores porcentuales)

\begin{tabular}{lcr}
\hline Relación de Parentesco & Consumo excesivo de alcohol & Consumo de droga \\
\hline Padre & 13,0 & 2,3 \\
Madre & 1,8 & 0,3 \\
Hermana/o & 2,8 & 0,8 \\
Otro/a pariente & 22,0 & 5,4
\end{tabular}

\subsection{Prevalencia y edad de inicio:}

De conformidad con lo expuesto en la tabla 4, se observan importantes niveles de consumo de sustancias en la población estudiada. Asimismo, las proporciones de haber experimentado el ofrecimiento de alguna droga también pueden interpretarse como elevadas, sobre todo al considerar el promedio de edad del grupo.

En efecto, existe una relación significativa entre el ofrecimiento y el consumo de drogas $(p<0.000)$, donde del $58,4 \%$ de los estudiantes que han recibido oferta, un $12,5 \%$ han consumido. Asimismo, llama la atención que, en el caso del alcohol, la proporción de haber experimentado su oferta sea menor que la respectiva prevalencia de vida de consumo; esto podría obedecer entre otros factores a patrones socioculturales vinculados con la ingestión de bebidas alcohólicas (por ejemplo, la disponibilidad de alcohol en los hogares y la asociación entre el consumo de alcohol y festividades religiosas y familiares) y con un fácil acceso 
Tabla 4. Prevalencia de consumo de sustancias y oferta de drogas en estudiantes de $5^{\circ}$ y $6^{\circ}$ grados de escuelas urbano-marginales, por tipo de droga, según categoría de prevalencia y oferta:

Heredia, Costa Rica, 1998. (Valores porcentuales)

\begin{tabular}{lccccc}
\hline Tipo de Droga & $\begin{array}{c}\text { Oferta de } \\
\text { Drogas }\end{array}$ & $\begin{array}{c}\text { Prevalencia } \\
\text { de vida }\end{array}$ & $\begin{array}{c}\text { Prevalencia } \\
\text { Último año }\end{array}$ & $\begin{array}{c}\text { Prevalencia } \\
\text { Último mes }\end{array}$ & $\begin{array}{c}\text { Prevalencia } \\
\text { Última semana }\end{array}$ \\
\hline Tabaco & 22,8 & 21,5 & 12,4 & 7,5 & 4,4 \\
Alcohol & 10,6 & 16,6 & 8,7 & 5,9 & 2,8 \\
Cocaína & 1,0 & 0,8 & 0,5 & 0,3 & 0,3 \\
"Crack" & 1,6 & 1,0 & 0,5 & 0,3 & 0,3 \\
Mariguana & 4,4 & 1,6 & 0,8 & 0,5 & 0,5 \\
\hline
\end{tabular}

a la adquisición de éstas, a pesar de ciertas normas jurídicas que prohiben su venta a menores de edad.

Al igual que lo sucedido en varias investigaciones de carácter nacional con diversos tipos de población (Bejarano, Amador y Vargas, 1994; Bejarano et al., 1995; Bejarano, Carvajal y San Lee, 1996; Sandí et al., 1995), las sustancias legales fueron las más consumidas, denotándose diferencias porcentuales marcadas con respecto a las drogas ilegales.

Lo anterior asume una severa connotación en el ámbito de la Salud Pública, al tomar en consideración el promedio de edad de la población bajo estudio (12,3 años), pues la legislación costarricense prohibe la venta de productos de tabaco ${ }^{2}$ y bebidas alcohólicas ${ }^{3}$ a personas menores de edad. Esta situación podría estar relacionada con el hecho que al $60 \%$ de las y los menores que han consumido drogas, le ofrecieran por primera vez la sustancia en un contexto hogareño (en su casa, la casa de un familiar, o la casa de un/a amigo/a).
Además se determinó que no existe una significancia en la relación entre el consumo más temprano y el mayor consumo activo de tabaco o fumado; sin embargo, es evidente en aquellos/as escolares que iniciaron el fumado entre los 11 y los 13 años de edad, una mayor tendencia a consumir otras drogas (72,3\%) no necesariamente ilegales.

En la figura 2 se muestran las edades promedio de primer consumo para las diferentes drogas, lo cual evidencia no sólo lo temprano del inicio en la ingesta, en una etapa del desarrollo humano en que se sientan las bases de la madurez adulta, sino que además, se constituye en un significativo factor de riesgo para diversas problemáticas psicosociales.

Se muestra una relación altamente significativa $(p<$ 0,000 ) entre la edad y el consumo de tabaco, a mayor edad mayor es la probabilidad de consumo. En este caso, el $92,8 \%$ de los niños entre 12 y 14 años de edad han consumido tabaco, contrarrestado al 7,2\% entre los 9 y 11 años de edad. Para el caso del consu-

Figura 2. Edad promedio de primer consumo de drogas en estudiantes de $5^{\circ}$ y $6^{\circ}$ grados por tipo de droga. Escuelas urbano-marginales de Heredia, Costa Rica, 1998.

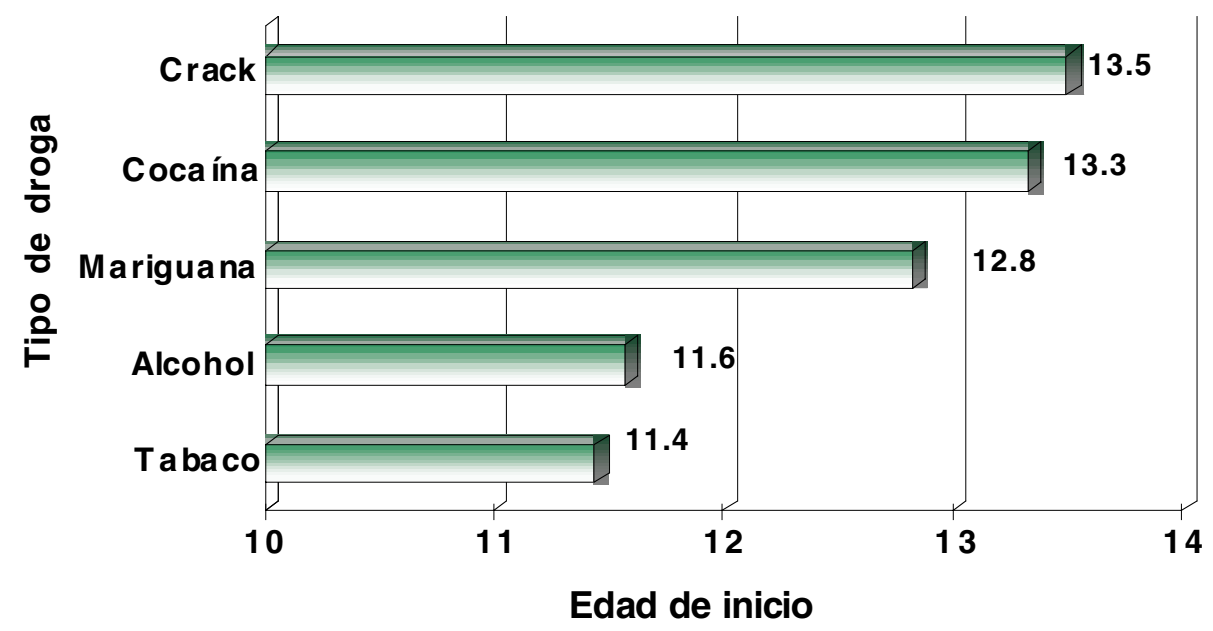

2 Ley N 7501 de 5 de mayo de 1995, "Ley de Regulación del Fumado" (artículo 4).

${ }^{3}$ Ley № 7633 de 21 de octubre de 1996, "Ley de Regulación de Horarios de Funcionamiento de Expendios de Bebidas Alcohólicas" (artículo 1). 
mo de alcohol, no se observa tal significancia, sin embargo, los porcentajes de consumo en los grupos de edad de los 9 a los 11 años $(12,5 \%$ ) y de los 12 a loa 14 años (87,5\%) discrepan lo suficiente como para mostrar una tendencia de mayor consumo a mayor edad.

A pesar que se ha considerado que el inicio temprano en el consumo de tabaco y alcohol puede conllevar un consumo posterior de otras drogas, en este estudio no se puede concluir en tal sentido, dadas las características etarias y académicas de la población estudiada.

\subsection{Factores asociados con el consumo:}

En la tabla 5 se presentan las principales razones que los y las estudiantes brindaron para no consumir drogas.

El hecho que una abrumadora mayoría indicara como principal razón para no consumir drogas que éstas causen daño a la salud, resulta un elemento de suma importancia a considerar por parte de los programas de prevención específica, sin caer en campañas informativas de corte médico-sanitario que privilegien la intimidación. Además, que en un cuarto lugar aparezca la categoría correspondiente a las y los estudiantes que no pudieron dar una razón para abstenerse de la ingesta de sustancias, es un aspecto de relevancia epidemiológica, ya que estaría evidenciando un sector de estudiantes sensible para iniciar el consumo, pues carecen de esquemas cognitivos relevantes que favorezcan una actitud negativa hacia la droga.

Asimismo, existe una significativa tendencia $(p<0,000)$ a no consumir drogas en relación con el credo religioso que se practica. Por ejemplo, del $69,7 \%$ de los/as niños/as que consideran que el consumo de drogas es nocivo para la salud, un 64,7\% comparten el catolicismo como credo religioso y un $19,3 \%$ el cristianismo. De aquellos/as que no lo hacen por respeto a los padres, la mitad son católicos $(51,4 \%)$ y una cuarta parte son protestantes.

Tabla 5. Razón principal para no consumir drogas en estudiantes de $5^{\circ}$ y $6^{\circ}$ grados de escuelas urbano-marginales de Heredia: Heredia, Costa Rica, 1998.

\begin{tabular}{lcr}
\hline Razón & Frecuencia Absoluta & Frecuencia Rel \\
\hline Perjudicial para la salud & & \\
Respeto a padres & 269 & $69,7 \%$ \\
Temor a Dios & 35 & $9,1 \%$ \\
Miedo & 41 & $10,6 \%$ \\
Otra & 4 & $1,0 \%$ \\
No sabe & 9 & $2,3 \%$ \\
& 28 & $7,3 \%$
\end{tabular}

\subsection{Diferencias por género y grado escolar:}

Un hallazgo relevante es que existe una relación significativa entre el grado académico y el haber recibido la oferta de drogas en general, así como también entre el grado escolar y el consumo de éstas $(p<0,000)$, lo cual permite establecer como tendencia que conforme aumenta el grado escolar $y$, por ende, la edad, mayor es la oferta y el consumo.

Otro aspecto de sumo interés es que las proporciones de consumo de tabaco son similares entre mujeres y varones e inclusive, las mujeres reportan un mayor nivel de consumo general, reciente y activo de alcohol (ver tabla 6). Lo anterior resulta en una preocupación con respecto al sector poblacional femenino, por cuanto diversas investigaciones nacionales (Bejarano, Amador y Vargas, 1994; Bejarano, Carvajal y San Lee, 1996; Jiménez y Bejarano, 1991) han demostrado de manera constante un significativo mayor consumo en los varones, a pesar que si se ha establecido un cambio en los patrones de consumo de las mujeres.
En el caso de la población femenina adulta este tipo de fenómeno se ha vinculado, entre otros aspectos, con situaciones de violencia intrafamiliar (Bejarano, San Lee y Carvajal, 1999; Sáenz, 1997; Walker, 1990).

\section{DISCUSIÓN}

Un elemento que produce inquietud en la reflexión posterior a este estudio, es el debate entre la deseabilidad social en las respuestas de los y las estudiantes y las expectativas de los investigadores, principalmente influidas por el "terrorismo informativo" de los medios de difusión de masas (Carranza, 1994) y los consecuentes prejuicios y estereotipos que estos generan en torno a la realidad de las comunidades urbano-marginales.

Al respecto, es evidente la incongruencia al considerarse que, precisamente son las drogas legales (alcohol y tabaco) de las que los y las estudiantes han 


\section{Tabla 6. Prevalencia de consumo de sustancias en estudiantes de $5^{\circ}$ y $6^{\circ}$ grados de escuelas urbano- marginales, por tipo de droga, según categoría de prevalencia y sexo: Heredia, Costa Rica, 1998.}

(Valores porcentuales)

\begin{tabular}{|c|c|c|c|c|c|c|}
\hline \multirow[t]{2}{*}{ Prevalencia por tipo de droga } & \multicolumn{2}{|c|}{ Prevalencia de vida } & \multicolumn{2}{|c|}{ Prevalencia de último año } & \multicolumn{2}{|c|}{ Prevalencia de último mes } \\
\hline & Masc. & Fem. & Masc. & Fem. & Masc. & Fem. \\
\hline Tabaco & 22,2 & 20,7 & 12,6 & 12,2 & 7,6 & 7,4 \\
\hline Alcohol & 16,2 & 17,0 & 7,6 & 10,1 & 5,6 & 6,4 \\
\hline Cocaína & 1,5 & - & 1,0 & - & 0,5 & - \\
\hline "Crack" & 1,5 & 0.5 & 1,0 & - & 0,5 & - \\
\hline Mariguana & 2,0 & 1,1 & 1,5 & - & 1,0 & - \\
\hline
\end{tabular}

NOTA: — No se registró información.

escuchado más pero que, a la vez, estiman menos peligrosas. Así pues, el alcoholismo es percibido como el segundo problema en importancia de la comunidad; sin embargo, se le califica como una droga de menor peligrosidad en comparación con las drogas ilegales, a pesar de que se manifiesta un consumo relativamente alto de alcohol en los escolares.

Un elemento que llama a la preocupación, tanto para las autoridades educativas como sanitarias y policiales es que el consumo de sustancias ilegales puede considerarse muy elevado en comparación con el estudio nacional sobre consumo de drogas de 1995, pues mientras que en la presente investigación, efectuada con población infantil, se hallaron prevalencias de vida de consumo de 1,6\% para mariguana, 0,8\% para cocaína y 1,0\% para "crack", en la población general estas proporciones fueron de 3,9\%, $0,9 \%$ y $0,4 \%$ respectivamente (Bejarano, Carvajal y San Lee, 1996). La magnitud de la severidad de esta situación no sólo estriba en la relativa similitud de las frecuencias en lo que a prevalencia de vida respecta, sino también en que el consumo de "crack" reportado por los y las estudiantes supera en más del 100\% la cifra del estudio nacional.

Además, para las tres drogas ilegales las prevalencias del último año y último mes de consumo fueron superiores en la población estudiada con respecto a la población nacional. Llama la atención que este fenómeno también se haya presentado en otras poblaciones vulnerables, tal como es el caso de las personas recluidas en el sistema penitenciario e ingresadas en salas de emergencia de hospitales generales (Bejarano, Sáenz y Ugalde, 1997; Bejarano y Sáenz, 1999; Sáenz y Bejarano, 1996; Sáenz et al., 1998).

En este sentido, resulta interesante que el consumo de "crack" reportado por los y las estudiantes supere el nivel de consumo de cocaína, lo que podría obedecer parcialmente a dos razones: a. menor costo económico (más barato) y b. un efecto psicofisiológico a más corto plazo. Esta tendencia también ha sido observada en otras poblaciones en que predominan características de vulnerabilidad psicosocial. (Bejarano, Sáenz y Ugalde, 1997; Sáenz y Bejarano, 1996; Sáenz et al., 1998).

Otro aspecto de suma relevancia epidemiológica es la temprana edad de inicio en el consumo, la cual se puede asociar con el intervalo de edad en que oscila la población estudiada, ya que existen significativas diferencias con respecto a la edad promedio de primer consumo en 1995 para la población de 12 a 70 años; situación que se ha relacionado tradicionalmente con el posterior consumo de drogas ilegales ("duras").

Asimismo, cabe destacarse en este acápite la similitud en los niveles de consumo de drogas legales, reportados según el sexo. Específicamente en el caso del alcohol y en relación con los planteamientos sobre el consumo de esta sustancia por parte de la población femenina adulta, detallados en párrafos precedentes, podría sugerirse como hipótesis para estudios posteriores que en la base del consumo reportado por las estudiantes existen situaciones de abuso intrafamiliar. Esta hipótesis se refuerza con datos de Stanton, Todd y colaboradores (1988) donde se señala que las mujeres adictas registran tasas de incesto mayores que lo normal, y con Walker (1990) quien refiere que la violencia doméstica es más significativa que la historia familiar en la predicción de abuso de sustancias.

En el ámbito de la prevención, se determinaron como factores de riesgo para el consumo de drogas: la disponibilidad de éstas (oferta), el consumo en el grupo familiar, así como una mayor edad y grado escolar. Sobresale como factor protector el mantener la práctica de algún credo religioso, aspecto éste que reiteradamente ha sido mencionado por los especialistas.

Los factores de riesgo identificados unidos a las características sociodemográficas propias de la población en estudio, permiten postularla a corto plazo, como una población de alto riesgo en relación con elevados niveles de consumo de drogas legales e ilegales. 
Lo anterior determina a esta población como prioridad en materia de prevención. Esto se traduce, primero, en un reto para las autoridades educativas del país en términos de adaptar el currículum educativo a la prevención, en tanto un estilo de vida que incorpore a la comunidad educativa. Y segundo, será necesaria la transformación y adecuación de políticas sociales en las comunidades marginales, dirigidas a cubrir carencias ligadas al desarrollo de problemas psicosociales.

\section{BIBLIOGRAFÍA}

Alfaro Mena, Olga; Cerdas Quirós, Douglas e Ibarra Cerdas, Marta. (1992). Estudio sobre menores trabajadores en la calle y con conductas de deambulación en Costa Rica, San José, Costa Rica: Centro Nacional para el Desarrollo de la Mujer y la Familia - Patronato Nacional de la Infancia (PANI).

Bejarano Orozco, Julio; Amador Muñoz, Giselle y Vargas Jiménez, Ana Lorena (1994). Consumo de drogas y percepciones de riesgo en el estudiante costarricense de $10^{\circ}$ y $11^{\circ}$ años 1993. San José, Costa Rica: Instituto sobre Alcoholismo y Farmacodependencia (IAFA).

Bejarano Orozco, Julio; Carvajal Morera, Hannia y San Lee Chacón, Lizú (1996). Consumo de drogas en Costa Rica: Resultados de la Encuesta Nacional de 1995. San José, Costa Rica: Instituto sobre Alcoholismo y Farmacodependencia (IAFA).

Bejarano Orozco, Julio; Sáenz Rojas, Mario A. y Ugalde Montero, Federico (1197). Consumo de drogas en sitios centinela: Costa Rica, 1996. (Quinta Ronda). San José, Costa Rica: Instituto sobre Alcoholismo y Farmacodependencia (IAFA) - Organización de Estados Americanos (CICAD/O.E.A.).

Bejarano Orozco, Julio; San Lee Chacón, Lizú y Carvajal Morera, Hannia (1999). “El consumo de drogas en la mujer costarricense. Evolución de cinco años"; Salud Mental -Instituto Mexicano de Psiquiatría- 22 (2): 41-48, abril.

Bejarano Orozco, Julio y Sáenz Rojas, Mario A. (1999). “Consumo de drogas en personas ingresadas en la Sala de Emergencias del Hospital San Juan de Dios: 19921996"; Revista Costarricense de Salud Pública -Asociación Costarricense de Salud Pública- (14): 20-31, jul.

Carranza Lucero, Elías (1994). Criminalidad: ¿Prevención o promoción?. San José, Costa Rica: Editorial de la Universidad Estatal a Distancia.

Guzmán, E. et al. (1982). La familia del menor inhalador y la intervención del trabajador social. San José, Costa Rica: Tesis en Trabajo Social, Universidad de Costa Rica (U.C.R.).

Herrera, Marvin y Valerio, Efraín. (1977). Investigación sobre alcohol y alcoholismo. III Ciclo de Enseñanza
Diversificada. San José, Costa Rica: Instituto Nacional sobre Alcoholismo (INSA).

Jiménez Martén, Francisco y Bejarano Orozco, Julio (1991). Estudio Nacional sobre Consumo de Alcohol y Drogas Ilícitas 1990. San José, Costa Rica: Instituto sobre Alcoholismo y Farmacodependencia (IAFA).

Míguez, Hugo (1983). Detenciones y drogas. San José, Costa Rica: Instituto Nacional sobre Alcoholismo (INSA).

Míguez, Hugo (1984). Estudio de menores inhalantes en una comunidad marginal. San José, Costa Rica: Instituto Nacional sobre Alcoholismo (INSA).

Míguez, Hugo, Bolaños, Dennis y Alfaro, Ana. (1985). Actitud del estudiante hacia el alcohol y las drogas. Perfil urbano-rural. San José, Costa Rica: Instituto Nacional sobre Alcoholismo (INSA).

Obando Solano, Patricia, Bejarano Orozco, Julio y Ugalde Montero, Federico (1998). Estudio sobre opiniones y consumo de sustancias psicoactivas en estudiantes de 5 to y 6 to grados de Enseñanza Básica de la Provincia de Limón. Proyecto Piloto de Prevención del Consumo de Drogas para la Costa Atlántica de Centroamérica. Informe, Mayo 1998. San José, Costa Rica: Instituto sobre Alcoholismo y Farmacodependencia (IAFA)-Organización de Estados Americanos (CICAD/O.E.A.).

Sáenz Rojas, Mario Alberto (1997). "Alcohol y alcoholismo: Un severo problema de Salud Pública en Costa Rica"; Revista de Ciencias Sociales -Universidad de Costa Rica- (77): 21-34, setiembre.

Sáenz Rojas, Mario A. y Bejarano Orozco, Julio (1996). "Consumo de drogas en el Área Metropolitana de San José: Resultados preliminares a partir de un Sistema de Vigilancia Epidemiológica (1992- 1994); Revista de Ciencias Sociales -Universidad de Costa Roca, (73- 74): 716, setiembre-diciembre.

Sáenz Rojas, Mario A., Bejarano Orozco, Julio, Alvarado Cordero, Ronald y Briceño Barahona, Gustavo (1998). "Privados de libertad y drogas: Experiencias en un régimen de confianza"; Medicina Legal de Costa Rica-Asociación Costarricense de Medicina Forense- 15 (1-2):6268, mayo-diciembre.

Sandí Esquivel, Luis Eduardo et al. (1995). Adolescencia y consumo de drogas en Costa Rica. Heredia, Costa Rica: Instituto sobre Alcoholismo y Farmacodependencia (IAFA) - Universidad Nacional (UNA).

Stanton, M. Duncan; Todd, Thomas C. y colaboradores (1988). Terapia familiar del abuso y adicción a las drogas. Traducción de Carlos Gardini. Buenos Aires, Argentina: Editorial Gedisa.

Walker, Leonore (1990). Las madres agredidas, los niños y el abuso de sustancias. San José, Costa Rica: Instituto Latinoamericano de las Naciones Unidas para la Prevención del Delito y el Tratamiento del Delincuente (ILANUD). 
